NOTAS Y EXPERIENCIAS/ NOTES AND EXPERIENCES

\title{
Acceso a datos de investigación e información científica en Chile
}

\author{
Patricio Andrés Padilla Navarro*, Osvaldo José Curaqueo Pichihueche*, \\ Ronald Domingo Cancino Salas**, Mario Hernán Gatica Álvarez*** \\ * Instituto de Desarrollo Local y Regional. Universidad de La Frontera, Temuco. Chile \\ ** Centro de Investigaciones Sociológicas. Universidad de La Frontera, Temuco. Chile \\ ***Dirección Académica de Pregrado, Universidad de La Frontera. Temuco. Chile \\ Correo-e: patricio.padilla@ufrontera.cl, osvaldo.curaqueo@ufrontera.cl, ronald.cancino@ufrontera.cl, mh.gatica@gmail.com
}

Recibido: 19-03-2012; 2a versión: 07-05-2012; Aceptado: 08-05-2012

Cómo citar este artículo/ Citation: Padilla-Navarro, P. A.; Curaqueo-Pichihueche, O. J.: Cancino-Salas, R. D.; Gatica-Álvarez, M. H. (2013). Acceso a datos de investigación e información científica en Chile. Revista Española de Documentación Científica, 36(3):en010. doi: http://dx.doi.org/10.3989/redc.2013.3.960

Resumen: En el siguiente artículo se presentan los principales análisis y resultados del primer estudio desarrollado en Chile para medir las capacidades en gestión de datos de investigación e información científica generada con fondos públicos. Los resultados se presentan de acuerdo a diez principios y directrices elaborados por la OCDE, para orientar a los países a tomar medidas que permitan materializar políticas y procedimientos de gestión estandarizados y consensuados entre los distintos actores involucrados en su generación.

Palabras clave: Datos de investigación; información científica; políticas científicas y tecnológicas.

\section{Access to research data and scientific information in Chile}

Abstract: The following article presents the principal results and analysis from the first study carried out in Chile designed to measure the capacity to manage the research data and scientific information created through public funding. The results are presented according to ten principles and guidelines prepared by the OECD, to guide countries towards taking actions that help bring about standardized management policies and procedures agreed among the different actors involved in their creation.

Keywords: Research data; scientific information; science and technology policies.

Copyright: () 2013 CSIC. Este es un artículo de acceso abierto distribuido bajo los términos de la licencia Creative Commons Attribution-Non Commercial (by-nc) Spain 3.0. 


\section{INTRODUCCIÓN}

La OCDE ha desarrollado directrices internacionales que orientan y regulan el acceso a datos de investigación e información científica generados con fondos públicos por investigadores e instituciones científico-tecnológicas: Apertura, Transparencia, Interoperabilidad, Profesionalismo, Calidad, Eficiencia, Sostenibilidad, Seguridad, Flexibilidad, Conformidad legal y Protección de la propiedad intelectual (Pilat y Fukasaku, 2007). Estos principios pretenden constituirse en estándares internacionales y así poder orientar a países en el diseño de políticas de gestión en el patrimonio de datos de investigación e información científica; el carácter patrimonial de los datos de investigación e información científica es adquirido por ser generados con ayuda total o parcial de fondos públicos, es decir, emanados desde el Estado mediante sus políticas e instrumentos para la investigación y desarrollo desde cualquier nivel de gobierno.

Los datos de investigación se definen como registros de hechos (resultados numéricos, textos, imágenes y sonidos) utilizados como fuentes primarias para la investigación científica y comúnmente aceptados en la comunidad científica por permitir la validación de resultados en la investigación científica. Un set de datos de investigación constituye una representación sistemática parcial del objeto que está siendo investigado. La información científica refiere a las conclusiones obtenidas del análisis de datos y a los resultados de una investigación (CONICYT, 2010).

Los países miembros de la OCDE se encuentran desarrollando estudios de diagnóstico para conocer su realidad en la gestión de datos de investigación e información científica y así poder construir planes y políticas de mejora en la gestión atendiendo a las directrices señaladas anteriormente. Actualmente Chile se encuentra desarrollando una política nacional de gestión en este campo, proceso que se inserta en una serie de ajustes en el diseño institucional chileno para la gestión de la ciencia, tecnología e innovación (Cancino, 2009). El desarrollo de una política de gestión datos de investigación e información científica en Chile requiere, en primer lugar, un diagnóstico que permita conocer las condiciones en que se encuentra y así poder desplegar una política de gestión. Para la consecución de tal propósito se realizó un estudio denominado "Estado del Arte Nacional e Internacional Sobre Manejo y Políticas de Acceso a Datos de Investigación e Información Científica y Tecnológica Financiada con Fondos Públicos y Recomendaciones de Buenas Prácticas" (CONICYT, 2010). El estudio se concentró en dimensiones asociadas al desarrollo de innovaciones promovidas en Chile: institucionalidad, capital humano, infraestructura tecnológica, patrimonio, vinculación y políticas de gestión (CNIC, 2007). Constó con una primera etapa de benchmarking internacional en gestión de datos de Investigación e información científica, una segunda etapa donde se realizaron las capacidades chilenas en esta materia y finalmente una etapa de recomendaciones para la generación de políticas y protocolos.

En el presente artículo los resultados se presentarán con una diferencia del estudio original, ya que se analizará la realidad chilena en materia de gestión de datos de investigación e información científica, considerando las directrices generadas y promovidas por la OCDE:

Flexibilidad: directriz que aboga por la necesidad de considerar el contexto social, económico y legal cuando países e instituciones generan mecanismos de acceso a datos de investigación e información científica.

Conformidad legal: necesidad de respetar la legislación interna para la difusión de datos de investigación e información científica atendiendo a la seguridad nacional, privacidad, confidencialidad, secretos comerciales, derechos de propiedad intelectual, protección de especies raras, amenazadas o en peligro de extinción.

Rendición de cuentas: referido a las evaluaciones periódicas de los usuarios de datos de investigación e información científica.

Apertura y transparencia: requerimientos en la igualdad de condiciones para el acceso a datos de investigación e información científica, acceso que debe ser al menor costo posible en dinero y en buenas condiciones.

Interoperabilidad y profesionalismo: necesidad de institucionalizar las labores de gestión de datos de investigación e información científica.

Calidad y eficiencia: Corresponden a las directrices más importantes ya que apuntan a mejorar los procesos en las etapas secuenciales de la gestión de datos de investigación e información científica, lo que hace necesario estandarizar procedimientos de forma participativa con los investigadores y considerar las diferencias entre áreas y disciplinas científicas.

Sostenibilidad: Existencia de clausulas que permitan regular el tratamiento, distribución y petición del patrimonio.

Seguridad: Vela por garantizar la integridad, resguardo y protección de los datos de investigación e información científica pero no en un sentido legal, sino considerando su sobrevivencia y no exposición a ser dañados o eliminados.

De esta forma este artículo pretende dar a conocer la trayectoria que ha tenido Chile para alcanzar estándares internacionales en gestión de datos de investigación e información científica, identificando sus brechas y desafíos. 


\section{LA GESTIÓN DE DATOS DE INVESTIGACIÓN E INFORMACIÓN CIENTÍFICA EN CHILE}

La definición de una estrategia para abordar una adecuada gestión de los datos de investigación e información científica en Chile, considerando las directrices sugeridas por la OCDE, implica incorporar la experiencia internacional en esta materia, es decir, identificar los aprendizajes y buenas practicas que permitan adaptar e incorporar procedimientos que faciliten el transito chileno a una buena gestión del patrimonio de datos de investigación e información científica. Además, regular de mejor manera esta actividad es casi imperativo debido a que los datos de investigación e información científica generada en las instituciones chilenas son mayormente financiados con fondos públicos. Si bien lo anterior no equivale a pensar en un Estado con facultades de limitar el control que las mismas instituciones puedan hacer de su patrimonio, sí le confiere una mayor responsabilidad en el tipo de tratamiento que se realice.

El desarrollo de políticas de gestión a nivel internacional se caracteriza por la existencia de fuertes restricciones técnicas e institucionales en el acceso a datos de investigación e información científica (Den Besten y otros, 2010) y la necesidad de comenzar el desarrollo de políticas que fomenten el acceso abierto y la colaboración científica identificando problemas que existen para cada área científica en la gestión de datos e información (Rodríguez Gallardo, 2008) (San José Montano, 2009). Además de ello se refuerza la necesidad de generar un proceso de "sensibilización", es decir, dar a conocer e institucionalizar la problemática en todos los actores involucrados dando a entender lo vital de abordarla (Sabourin y Dumouchel, 2007).

En segundo lugar, se hace necesario la creación de organismos que resguarden los datos de algún área científica determinada y el desarrollo de inventarios de los activos de información del sector público (Ramos y otros, 2009) y procedimientos de estandarización de información (Eito, 2007), entendidos por la OCDE como interoperabilidad (Pilat y Fukasaku, 2007, p. 10 ). Por lo general se alimentan de los resultados emanados de proyectos, investigaciones generadas con fondos públicos y puestas a disposición de la comunidad científica. Esto en el contexto chileno, implicará definir el rol de las agencias publicas y sobre todo los institutos tecnológicos y/o la creación de agencias de gestión de datos de Investigación e información científica.

En tercer lugar, los fondos que permiten el apoyo, o la total realización de proyectos científicos, incluyen dentro de sus aspectos normativos no sólo lo relacionado con los objetivos globales de carácter estrictamente científico, sino además lo concerniente al tratamiento y accesibilidad de bases de datos e información más relacionada con procesos que con resultados. Ello, en el caso chileno, requiere ser desarrollado ya que las normativas actuales no contemplan estos aspectos.
Entre las directrices promovidas por la OCDE existen dos muy generales y una sintomática: flexibilidad y la conformidad legal y el demostrativo a la rendición de cuentas (Pilat; Fukasaku, 2007). Sin embargo, existen otras mucho más específicas que refieren a la apertura, transparencia, interoperabilidad, profesionalismo, calidad, eficiencia, sostenibilidad y seguridad. Éstas han sido estudiadas para el caso chileno y son presentadas a continuación,

\subsection{Apertura y transparencia}

Los principales aspectos analizados para conocer el estado de apertura y transparencia en las instituciones e investigadores que gestionan datos de investigación e información científica en Chile, fue, en primer, lugar el proceso de diseminación, entendido como la práctica de difundir el patrimonio de datos de investigación e información científica de forma activa, promoviendo el acceso a dicho patrimonio para otras instituciones o investigadores. En segundo lugar, se analizó la normativa de los fondos del Sistema Nacional de Innovación chileno en la dimensión de difusión de la información.

El indicador para medir las características de la diseminación fue el nivel de accesibilidad del patrimonio de datos de investigación e información científica, el que permite analizar el tipo de acceso o restricción que instituciones e investigadores ofrecen al patrimonio que generan (ver figura 1 ).

Los resultados muestran que no existe una gran diferencia entre la proporción de instituciones que dan acceso total y parcial al patrimonio de datos de investigación e información científica en Chile. Si bien existe un leve porcentaje mayor en las instituciones que dan acceso parcial, éste no es suficiente para señalar que aquella es una característica generalizada.

El estudio evidenció que el intercambio de patrimonio es mayoritariamente gratuito y sin preferencias a un sector en particular, ya que el traspaso se realiza tanto a actores del sector público, privado y académico. Sin embargo, algunas instituciones realizan cobros en dinero por ciertos datos de carácter estratégico, el que es concedido mediante la firma previa de convenios que resguarden una adecuada utilización.

El aspecto que impide a Chile constituirse como un país con apertura en su patrimonio de datos de investigación e información científica, es la inexistencia de instituciones e investigadores que realizan procesos de diseminación, si bien existen instituciones que consideran adecuado y ventajoso el intercambiar y difundir su patrimonio, estas prácticas no corresponden exactamente a lo que se entiende por diseminación. En este sentido, existe un rol más pasivo en la forma de relacionar al entorno con el patrimonio generado, debido a que la mayor fuente para difundir los datos de investigación e información científica es mediante el alojo de éstos en páginas web, procedimiento que no va acompa- 
ñado de servicios de alerta, boletines o algún medio que permita contactar a posibles instituciones $\mathrm{y}$ actores que puedan hacer un uso apropiado y pertinente de dicho patrimonio.

También se consultó a las instituciones el tipo de ventajas y desventajas que observan en el proceso de diseminación. Las primeras se relacionan con sus posibilidades en el ámbito científico, es decir, el potencial desarrollo de nuevas investigaciones y aplicaciones que permitirían el intercambio de patrimonio con otros agentes. Además, se identificaron ventajas relacionadas con evidenciar la transparencia en el gasto público, entendido como el explicitar mediante diseminación, la forma en que se han utilizado recursos económicos de carácter estatal. Por último, se señalan ventajas asociadas al vínculo con el entorno que generan las prácticas de diseminación, tanto para el sector privado, público y académico. Las desventajas de se relacionan con las aprehensiones sobre usos no adecuados al patrimonio por parte de terceros y la limitación de la competitividad científica.

Para conocer aún más el estado de apertura y transparencia en Chile, se analizó la normativa de los principales programas, proyectos y convocatorias de las instituciones que otorgan fondos para actividades de investigación, desarrollo e innovación que forman parte del Sistema Nacional de Innovación en Chile. El análisis se refirió específicamente a la difusión de información, analizando si los fondos establecen responsables o figuras de responsabilidad en la actividad de difusión, la existencia de cláusulas que permitan la libre difusión de información no confidencial y las relativas a la disposición de procedimientos de planificación de las actividades de difusión (ver figura 2).

Figura 1. Accesibilidad del patrimonio al entorno de instituciones que gestionan datos de investigación e información científica

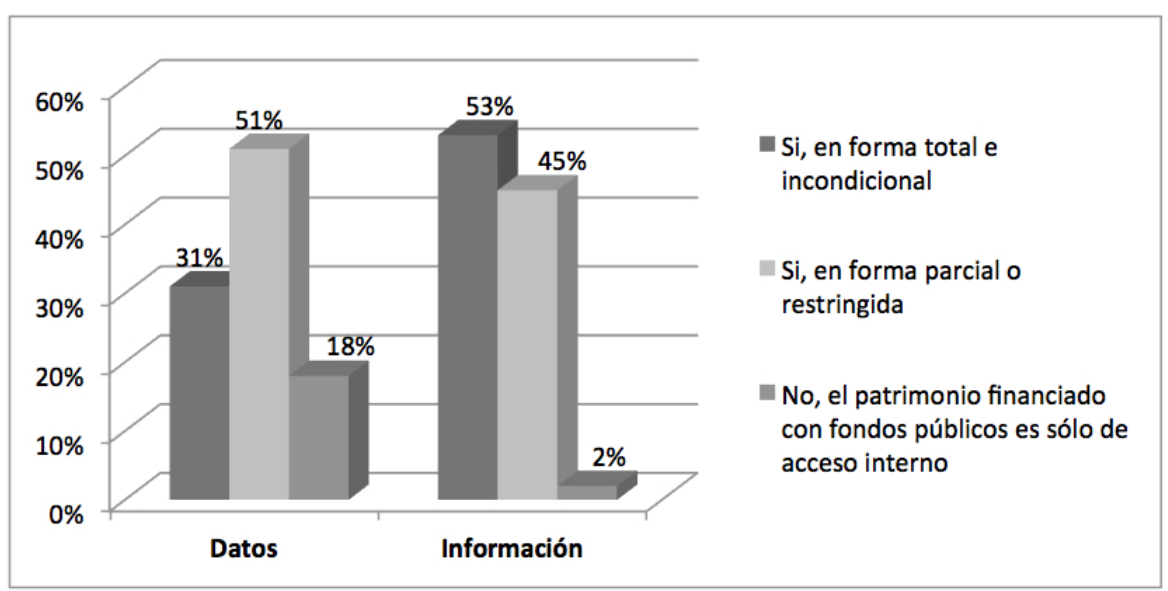

Fuente: elaboración propia, IDER, 2011.

Figura 2. Definición de la responsabilidad de las actividades de difusión

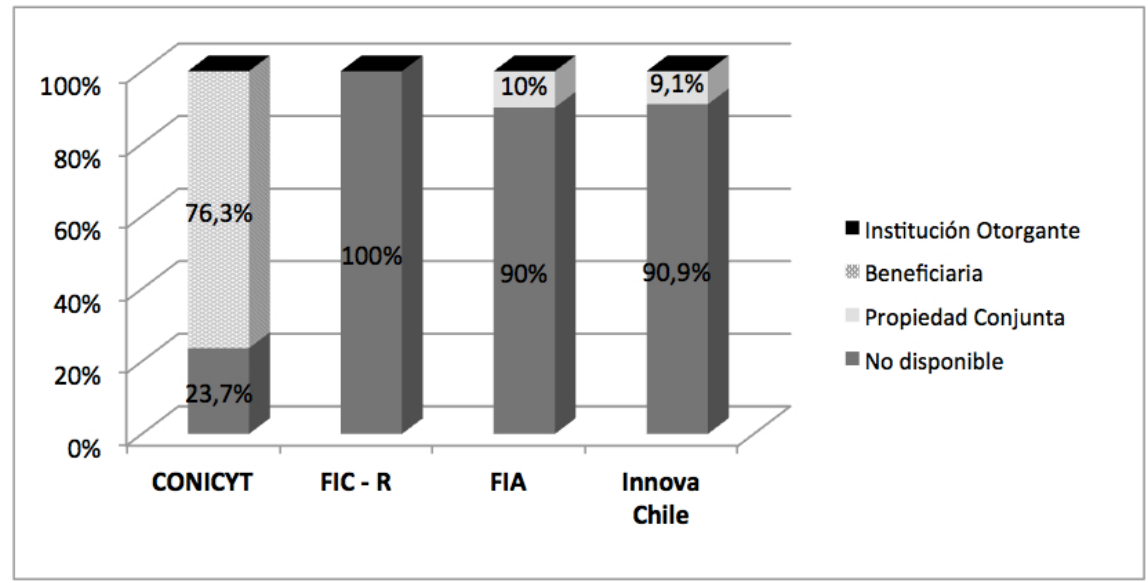

Fuente: elaboración propia, IDER, 2011. 
Los resultados evidenciaron que casi la totalidad de los fondos públicos no definen responsabilidades en la ejecución de actividades de difusión a excepción de la Comisión Nacional de Investigación Científica y Tecnológica de Chile, (en adelante CONICYT) quien reconoce en un grado significativo a la beneficiaria como responsable de estas actividades $(76,3 \%)$. En los Fondos de Innovación para la Competitividad de Asignación Regional (en adelante FIC-R) este aspecto no se encuentra disponible y en los demás fondos la definición de responsabilidad no supera el $10 \%$ además de establecerse una responsabilidad conjunta, la que si bien garantiza un compromiso por las partes, puede diluirse entre ellas en entornos de incertidumbre.

\subsection{Interoperabilidad y Profesionalismo}

Aunque la ciencia se está convirtiendo en una gran empresa mundial, la incompatibilidad de las normas técnicas y de procedimientos puede ser un serio obstáculo para los usos múltiples de los conjuntos de datos. Atendiendo a ello, la directriz de interoperabilidad refiere a la disposición de normas internacionales pertinentes y de uso común que faciliten el contacto de instituciones e investigadores al interior de un país y en cooperación con otras organizaciones internacionales (Pilat y Fukasaku, 2007 , p. 10). Para esto debe existir un contacto con los estándares y normas internacionales de documentación según áreas científicas, donde exista colaboración mediante convenios y redes nacionales e internacionales.

El profesionalismo implica la necesidad de desarrollar reglas institucionales para la gestión de datos de investigación e información científica basada en normas aplicables y sobre valores inscritos en los códigos de conducta de las comunidades científicas implicadas. Para ello es necesario utilizar procedimientos y reglas claras para la gestión de patrimonio además de generar un clima que permita la confianza entre los investigadores e instituciones. Lo anterior implica disponer de procesos de planificación en la gestión de datos de investigación e información científica, proceso llevado a cabo por expertos en estas tareas.

El análisis realizado en interoperabilidad y profesionalismo para el caso chileno evidenció que el mayor porcentaje de instituciones e investigadores que gestionan tanto datos de investigación como información científica lo hacen con ausencia de administradores de bases de datos especializados, sin protocolos para los procedimientos de respaldo, y con formatos manuales para el tratamiento de datos, escaseando métodos automáticos o más avanzados. Así mismo, se analizó el nivel de vínculos de instituciones e investigadores mediante su participación en redes, programas, o convenios de cooperación, que impliquen la posibilidad de compartir datos de investigación e información científica. Los resultados evidenciaron un alto nivel de presencia en redes internacionales de carác- ter mayormente académico y público. Además los encuestados valoraron positivamente estas redes para la actividad científica por parte de las instituciones.

Las razones esgrimidas por las instituciones que no comparten su patrimonio se refieren esencialmente a su diseño institucional: la vinculación no resulta ser una prioridad, no se dispone de capital humano pertinente y se argumenta la posibilidad de disminuir la competitividad científica de la institución que entrega sus datos de investigación e información científica.

\subsection{Calidad y Eficiencia}

La calidad y la eficiencia corresponden a las directrices más importantes ya que apuntan a mejorar los procesos en las etapas secuenciales de la gestión de datos de investigación e información científica, lo que hace necesario estandarizar procedimientos de forma participativa con los investigadores y considerar las diferencias entre áreas y disciplinas científicas.

Para medir la directriz de calidad y eficiencia en las instituciones chilenas se han considerado los niveles de centralidad y exclusividad en la gestión del patrimonio y la existencia de políticas que estandaricen los procedimientos de gestión. La centralidad se refiere al grado de concentración que poseen las labores de gestión de datos e información, si las instituciones poseen un departamento, unidad o profesional dedicado solamente a este tipo de funciones implica que efectivamente posee centralidad. La exclusividad se refiere a la cantidad de tareas relacionadas con la gestión de datos de investigación e información científica respecto a tareas de naturaleza distinta, por ejemplo, en el caso de que las instituciones tengan como quehacer solamente la gestión de datos de investigación y/o información científica quiere decir que poseen exclusividad.

Un porcentaje altísimo de instituciones chilenas que gestionan datos de investigación y/o información científica, lo hacen carentes de unidades especializadas en estas prácticas traspasando esas responsabilidades a los propios investigadores, lo que expresa un bajo nivel de centralidad en las funciones de gestión. El nivel de exclusividad también es bajo ya que un porcentaje minoritario de instituciones se dedica únicamente a labores de gestión de datos de investigación e información científica. En Chile existe una baja formalización de estas labores en las instituciones estudiadas donde cada uno de los investigadores efectúa procedimientos de gestión de forma autónoma y desorganizada, resolviendo problemas según sus propios conocimientos y necesidades

Los resultados en calidad y eficiencia evidencian una clara brecha entre la realidad nacional y las buenas prácticas ya que la mayor parte de las instituciones que gestionan datos de investigación e in- 
formación científica no poseen políticas de gestión y en otras sus funcionarios no saben de su existencia. Respecto a las instituciones que sí poseen políticas de gestión para regular sus procedimientos, el nivel de aplicación que poseen es sólo por algunas personas y además son sólo indicativas, por lo que existe una ausencia de protocolos, manuales de operaciones y niveles de formalización básicos con que gestores y profesionales, al interior de las instituciones, puedan operar en relación a estas materias.

\subsection{Sostenibilidad y Seguridad}

Los aspectos posteriores a la generación de datos de investigación e información científica se relacionan con la forma en que son utilizados, se comparten y protegen. La sostenibilidad se refiere a la existencia de clausulas que permitan regular el tratamiento, distribución y petición del patrimonio (Pilat y Fukasaku, 2007, p. 11). La seguridad vela por garantizar la integridad, resguardo y protección de los datos de investigación e información científica pero no en un sentido legal, sino considerando su supervivencia y no exposición a ser dañados o eliminados, en particular mediante el apoyo a la utilización de técnicas e instrumentos apropiados, incorporando seguros contra un daño o pérdida intencional o no intencional, daños ambientales y acceso no autorizado.

El estado de seguridad se relaciona con la existencia en las instituciones científicas de procedimientos que contemplen el resguardo del patrimonio generado y las características del soporte donde se realiza dicho respaldo junto a su frecuencia, lo que supone la existencia de protocolos e infraestructura asociada para respaldar el patrimonio. El caso chileno evidenció que las instituciones que gestionan datos de investigación e información científica disponen en su mayoría de prácticas de respaldo. Además, los soportes institucionales, es decir, los medios privilegiados para el almacenamiento de datos de investigación e información científica son unidades ópticas por su bajo precio y alta capacidad de almacenamiento, pero la seguridad de los respaldos en este tipo de medios es más susceptible a extravíos y/o deterioro a causa de la humedad o el paso del tiempo. En cambio, otros medios como los magnéticos (discos duros externos y sobre todo servidores especializados) otorgan más seguridad pero a un mayor costo de implementación. De acuerdo a las instituciones encuestadas, la mayor parte de las que manejan datos de investigación e información científica prefieren el respaldo en servidores propios.

Para conocer las características de la sostenibilidad se analizaron las normativas de los fondos públicos del Sistema Nacional de Innovación en Chile pero en base a la dimensión del data sharing, es decir, modalidades y características en la entrega y distribución de datos, pensando en la definición por clausulas para un soporte o formato específico en la entrega de información, clausulas referidas al tipo de acceso a que se hará del patrimonio y el referente al cumplimiento de entrega de la información.

Respecto a la definición del soporte y/o formato de los productos resultantes de las actividades seleccionadas en la concesión de los fondos, los resultados fueron diferenciados ya que si bien existen programas que en un alto grado hacen solicitud expresa de esta formalidad, existen otros en los cuales la mitad de los fondos analizados no cumplen con este aspecto. De forma concreta, InnovaChile y CONICYT, con un $95,5 \%$ y un $68,8 \%$ respectivamente, muestran los mejores resultados. Por el contrario, FIA en sólo un $40 \%$ de sus programas analizados hace mención a este requisito, y con el peor resultado se identifica al programa FIC-R, el cual en la totalidad de sus fondos carece de esta solicitud.

\section{CONCLUSIONES}

Del estudio realizado es posible extraer al menos tres aprendizajes relevantes para el caso chileno. En primer lugar la necesidad de hacer patente el carácter público de los datos de investigación e información científica financiados con fondos estatales y su importancia para el desarrollo y la competitividad. En el caso de las Políticas de Intercambio de Datos Científicos en China evidenció la dificultad de desarrollar una política nacional de gestión cuando en la cultura científica local persiste la idea de propiedad personal de los investigadores de los datos de investigación e información científica financiados con fondos públicos. Para esto se realizó una campaña que puso en valor la necesidad de desarrollar políticas de gestión de datos como soporte para el desarrollo del país y su competitividad (Xu, 2007).

Se hace necesario instalar la discusión respecto a cómo entender lo público y cómo los distintos fondos, además de ayudar a promover el desarrollo científico, están pensados y orientados al desarrollo de los países, lo que implica no sólo cumplir con los objetivos planteados en la fase de postulación de los proyectos de investigación y estudios aplicados, sino además, incorporar en el acervo de productos comprometidos la parte intermedia relacionada con los procesos como lo son las bases de datos y el levantamiento de información, ya que éstos son vitales para el avance de iniciativas posteriores (Uhlir y Schröder, 2007).

En segundo lugar, la necesidad de resguardo de datos de investigación e información científica de trato especial: existe cierta información, que si bien no puede ser diseminada de forma abierta a todas las instituciones por el mal uso que puede hacerse de ella, es necesario construir mecanismos que permitan darle algún tipo de diseminación que sea beneficioso y útil (Schröder, 2007) (Abowd y Lane, 2004) (Bachrach y Spittel, 2011). Esto su- 
cede con los datos epidemiológicos del National Center for Health Statistics en Estados Unidos, debido a lo delicado de la información manipulada, se construyó un "seguro de investigación" llamado RDC, en éste el almacenamiento responde a determinados criterios y se permite el ingreso bajo una estricta supervisión. La única forma de acceder al RDC es mediante una propuesta de los investigadores que permita aceptarlos y hacer uso de este material. Ejemplos de restricción del RDC son la configuración especial de los puertos USB y todo tipo de mecanismos de transferencia de datos que lo hacen inaccesible. Las estaciones de trabajo se han configurado de tal manera que los usuarios reciban acceso de sólo lectura a datos y archivos solicitados, pudiendo escribir sólo en la estación de trabajo local del disco duro y así impedir que los usuarios eliminen información exenta de revisiones de confidencialidad. En este sentido, la información de difícil gestión, con características que la hacen diferentes y merecedoras de protocolos especiales, deben ser identificadas en las áreas científicas, establecer los motivos de resguardo y generar el tipo de restricciones y condiciones de acceso para su uso.

Por último se hace patente el requerimiento de promover la transdisciplinariedad en la gestión de datos de investigación e información científica. La gestión de datos de investigación e información científica no posee una disciplina o campo científico por excelencia de su propiedad, en ella intervienen gestores de información, ingenieros informáticos, lingüistas, bibliotecólogos, entre otros. En consecuencia, para establecer políticas nacionales de gestión es necesario disponer además de este tipo de profesionales, investigadores y académicos que entiendan y conozcan en detalle la estructura del campo científico en que se trabajará en una política; esto unido a que cierta información merece una decodificación distinta, tiene una composición más cualitativa que podría requerir tanto de habilidades informáticas y técnicas para su procesamiento en grandes bases de datos que interactúen, pero además, un conocimiento acabado de cómo esta información es procesada, relacionada y tipificada para que en el proceso de su almacenamiento y diseminación tenga una utilidad en los investigadores que la utilicen.

\section{BIBLIOGRAFÍA}

Abowd, J.; Lane, J. (2004). New Approaches to Confidentiality Protection: Synthetic Data, Remote Access and Research Data Centers Privacy in Statistical Databases. En: Domingo-Ferrar, J; Torra, B. (editores) Privacy in Statistical Databases. Springer-Verlag, New York.
Bachrach, C.; Spittel, M. (2011). Developing a Plan for Data Sharing. How to Write a Successful Research Grant Application. En: Stover, E; Anne Boyce, C. (editores) Willo Pequegnat. Springer, EE.UU.

Cancino, R. (2009). Sistemas Regionales de Innovación en Chile: estado actual y escenarios de futuro. En: Von Baer, E (Editor), Pensando Chile desde sus regiones. Ediciones Universidad de la Frontera. Temuco, Chile.

CNIC. (2007). Estrategia Nacional de Innovación - VoIumen I. Santiago: Gobierno de Chile. http://www. cnic.cl/content/view/468181/Hacia-una-Estrategia.html.

CONICYT, IDER. (2010). Estado del Arte Nacional e Internacional Sobre Manejo y Políticas de Acceso a Datos de Investigación e Información Científica y Tecnológica Financiada con Fondos Públicos y Recomendaciones de Buena Prácticas. Santiago, Chile.

Den Besten, M.; David, P.; Schroeder, R. (2010). Research in e-Science and Open Access to Data and Information. International Handbook of Internet Research. En: Hunsinger, J; Klastrup, L; Allen, M. (editores.) International Handbook of Internet Research. Springer, EE.UU.

Eito Brun, R. (2007). Normalización documental y colaboración electrónica: una visión retrospectiva. Revista Española de Documentación Científica, Vol. 30 (1), 24-48.

Pilat, D.; Fukasaku, Y. (2007). OECD Principles and Guidelines for Access to Research Data from Public Funding. Data Science Journal, Vol. 17, 4-11.

Ramos Simón, L.F.; Mendo Carmona, C.; Arquero Avilés, R. (2009). La producción informativa y documental del Estado: hacia un inventario de los recursos públicos. Revista Española de Documentación Científica, Vol. 32 (1), 40-59.

Rodríguez Gallardo, A. (2008). Elementos que fundamentan el Acceso Abierto. Investigación bibliotecológica, Vol. 22 (44), 161-182.

Sabourin, M.; Dumouchel, B. (2007). Canadian national consultation on access to scientific research data. Data Science Journal, Vol. 6, 26-35.

San José Montano, B. (2009). Acceso abierto (open access), un modelo necesario de comunicación científica. Pediatría Atención Primaria, Vol. 11 (42), p. 299-311.

Schröder, P. (2007). Possible Downsides to Data Sharing in the Research Commons: Assets and Liabilities, Opportunities and Risks. Data Science Journal, Vol. 6, 67-70.

Uhlir, P.; Schröder, P. (2007). Open data for a global science. Data Science Journal, 6, p. 36-53.

Xu, G. (2007). Open Access to Scientific Data: Promoting Science and Innovation. Data Science Journal, Vol. 6, 21-25. 